\title{
Modulation de la qualité de la viande de porc par l'alimentation
}

\author{
J. MOUROT, B. LEBRET
}

INRA, Agrocampus, UMR1079 Systèmes d'Elevage, Nutrition Animale et Humaine, F-35590 Saint-Gilles, France

Courriel : Jacques.Mourot@rennes.inra.fr

Les conditions d'élevage modifient les performances de croissance et la qualité des produits animaux. On peut distinguer les facteurs génétiques, ceux liés à la conduite d'élevage et à l'alimentation. Concernant les facteurs génétiques, le génotype des animaux aux locus de gènes à effet majeur sur la qualité : $H A L$ (gène de sensibilité à l'halothane dont l'allèle $H A L^{n}$ est responsable du défaut PSE ou «viandes pisseuses») et $R N$ (gène « Rendement Napole» dont l'allèle $R N^{-}$conduit aux viandes acides : à bas $\mathrm{pH}$ ultime), va déterminer les propriétés qualitatives des viandes. Par ailleurs, le type génétique influence fortement les performances de croissance et la composition de la carcasse et des tissus à l'abattage, avec pour conséquence un fort impact sur les qualités sensorielles, nutritionnelles et technologiques des viandes. La conduite d'élevage, notamment le type d'habitat (type de sol, accès à l'extérieur ou plein air) en interaction avec les conditions climatiques, influence également les performances de croissance, la composition corporelle et tissulaire, et la qualité des produits.

L'alimentation, c'est-à-dire le niveau de la ration, les conditions de distribution (restriction-réalimentation), l'équilibre entre les principaux nutriments et la nature de certains nutriments (lipides notamment) déterminent, pour un potentiel génétique donné, la vitesse et la nature du gain de poids donc la composition des tissus à l'abattage. Ainsi, parmi les facteurs d'élevage, l'alimentation joue un rôle central dans la construction de la qualité des viandes et produits du porc, et plus particulièrement sur les dimensions sensorielles et nutritionnelles des produits.

\section{1 / La viande de porc : prin- cipales caractéristiques}

Les propriétés des carcasses et des viandes de porc ont beaucoup évolué au cours des 40 dernières années suite aux programmes de sélection génétique basés sur l'augmentation de la vitesse de croissance et la réduction de l'épaisseur du tissu adipeux sous-cutané dorsal. La meilleure connaissance des besoins alimentaires des animaux, tout comme celle de la valeur nutritionnelle des matières premières, a été aussi un élément très positif dans cette évolution. L'adiposité globale de la carcasse a ainsi diminué de moitié au cours de cette période, la proportion de tissus adipeux passant de plus 40 à environ $20 \%$ du poids de la carcasse, au profit des tissus maigres (Lebret 2004). Cette évolution s'est accompagnée d'une réduction de la teneur en lipides totaux des tissus adipeux (de 5 à $10 \%$ pour le tissu adipeux du dos).

L'avantage de la viande de porc est que, lorsqu'elle est dans l'assiette du consommateur, ce dernier peut séparer facilement le gras visible de la viande. Et contrairement aux idées reçues, et malheureusement tenaces, la viande de porc est relativement maigre lorsque le gras visible est écarté. De nombreuses études ont montré que le muscle Longissimus, lorsqu'il est paré, contient moins de $2 \%$ de lipides. Or, une association positive entre la teneur en lipides et la qualité sensorielle, notamment la tendreté et la jutosité, a clairement été établie par des jurys d'experts ainsi que par des consommateurs. Une teneur d'environ 2,5 à $3 \%$ de lipides apparaît optimale (Fernandez et al 1999a et b, Brewer et al 2001). Bien évidemment, comme pour toutes les espèces animales, la teneur en lipides du muscle dépendra de la localisation anatomique (Culioli et al 2003). Pour la côte de porc entière, c'est-à-dire incluant les gras externe et intermusculaire, la teneur en lipides est voisine de 10 à $12 \%$, mais déjà au niveau de l'étal, le boucher peut choisir de diminuer l'épaisseur de gras externe. La teneur en lipides peut alors être inférieure à 5\%. Par ailleurs, la viande est une source majeure de protéines (17 à 20\% selon les morceaux), de minéraux et de certaines vitamines ( $\mathrm{B}_{12}$ en particulier).

Le porc constitue la principale viande consommée en France (34 kg/an/habitant) et en Europe (43 kg/an/h). En France, la consommation de viande de porc fraîche représente $30 \%$ de la production; l'essentiel de la viande de porc produite est donc consommé après transformation des viandes en produits crus ou cuits. La teneur en lipides des produits dépendra donc du mode de fabrication (2 à $15 \%$ de lipides pour une tranche de jambon et de 20 à $30 \%$ pour un pâté) (Mourot et al 2007). Les enquêtes INCA (Volatier 2000) ont montré que, parmi les produits animaux, les produits du porc sont les seconds fournisseurs de lipides dans l'alimentation humaine en apportant $11,7 \mathrm{~g}$ de lipides par jour, loin derrière les produits laitiers avec un apport voisin de $30 \mathrm{~g}$. L'importance de cet apport lipidique dans l'alimentation humaine justifie pleinement les recherches mises en place en France notamment par l'INRA pour améliorer à l'aide des facteurs d'élevage la valeur nutritionnelle de la fraction lipidique du porc pour le consommateur.

\section{2 / Effets du niveau alimen- taire et du profil de distri- bution sur la qualité de la viande}

\section{1 / Restriction alimentaire}

Une diminution des apports alimentaires relativement aux besoins des animaux (restriction) entraîne logiquement une réduction de la vitesse de croissance, proportionnelle à la réduction de l'ingéré. Chez l'animal en croissance, le dépôt de lipides étant plus sensible aux apports en énergie que le dépôt de protéines (Noblet 2005), la restriction alimentaire affectera plus fortement le dépôt de lipides 
par un effet de restriction énergétique. Cela entraîne une réduction de l'adiposité corporelle et de la teneur en lipides intramusculaires chez les animaux rationnés relativement à ceux alimentés à volonté (figure 1). Une altération des propriétés sensorielles des viandes peut alors être observée (Ellis et al 1996) mais n'est pas systématique car elle dépend notamment de la gamme de teneurs en lipides intramusculaires considérées (Lebret et al 2001). En terme de composition en acides gras, la moindre adiposité consécutive à la restriction énergétique résulte d'une réduction du taux de synthèse des acides gras, par nature saturés ou monoinsaturés chez le porc. Dans les lipides tissulaires, la part des acides gras d'origine exogène, c'est-àdire provenant de l'alimentation, polyinsaturés pour une grande part, est accrue : cela conduit à une augmentation du taux d'acides gras polyinsaturés dans les tissus adipeux des animaux restreints comparativement aux animaux alimentés à volonté (Wood et al 2008). Du point de vue nutritionnel, la restriction alimentaire est donc favorable tant sur la quantité des lipides que sur la nature de leurs acides gras constitutifs de la viande. Néanmoins, cette amélioration s'effectue sous réserve que le risque de peroxydation des acides gras polyinsaturés dans les produits soit en majorité prévenu par un apport d'antioxydants dans l'alimentation des animaux (cf. $\S 3.3$ ).

Enfin, les composantes de la qualité technologique de la viande (vitesse et amplitude de chute du $\mathrm{pH}$ post mortem, couleur, pertes en eau) ne sont pas influencées par la restriction alimentaire (Lebret 2008).

Figure 1. Stratégies alimentaires appliquées entre 30 et $110 \mathrm{~kg}$ de poids vif : alimentation à volonté $(A L)$, restriction alimentaire de $25 \%$ (R1), réduction progressive des apports en protéines relativement à l'énergie avec limitation des apports énergétiques (R2). Conséquences sur la teneur en viande maigre (TVM) des carcasses et le taux de lipides du muscle Longissimus (LIM) chez des porcs croisés Duroc ( ${ }^{* * *}: P<0,001$; les moyennes affectées d'une lettre différente diffèrent significativement, $P<0,05$ ). Adapté de Lebret et al (2001).
TVM, $\%$ ***

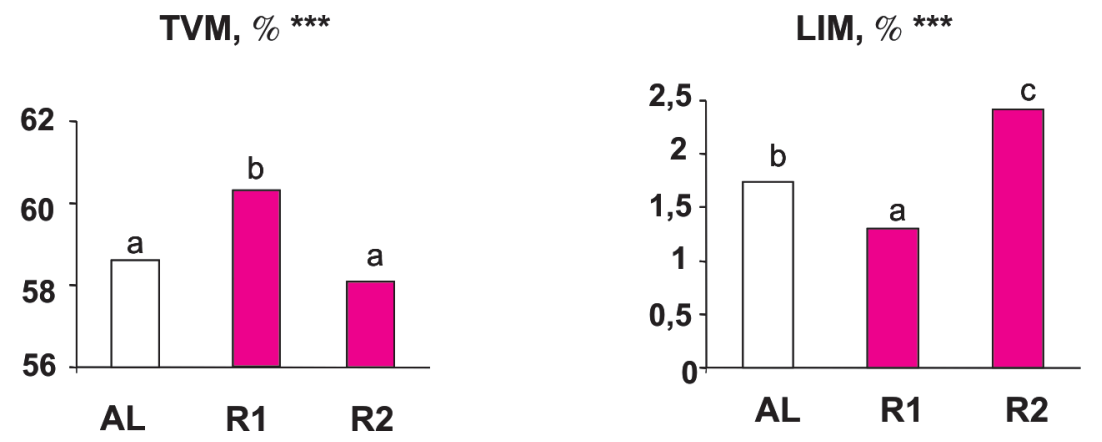

\section{2 / Restriction puis réalimen- tation : la croissance compensa- trice}

La croissance compensatrice est un phénomène physiologique d'accélération de la vitesse de croissance en finition. Elle est induite par une restriction alimentaire appliquée au cours de la première phase de croissance suivie d'une alimentation à volonté des animaux (et d'une consommation compensatrice). Le niveau de réponse de l'animal à cette stratégie alimentaire dépend du stade physiologique des animaux, de la durée et de l'intensité de la restriction alimentaire, ainsi que du stade et de la durée de la réalimentation (Lebret 2008). Au niveau corporel, la compensation en termes de vitesse et souvent d'efficacité de la croissance résulte d'une augmentation de la masse des dépôts adipeux et de la croissance des organes internes, mais pas d'une augmentation des dépôts musculaires. Ainsi, la restriction alimentaire réduit la masse des dépôts adipeux puis la réalimentation à volonté la favorise de nouveau. Au final, les animaux ayant exprimé une phase de croissance compensatrice présentent généralement une composition corporelle globale similaire à celle d'animaux alimentés à volonté pendant toute la croissance (Heyer et Lebret 2007). En revanche, au niveau musculaire, la restriction diminue certes le dépôt de lipides mais la réalimentation ultérieure ne s'accompagne généralement pas d'une augmentation de l'accrétion lipidique lorsque la réalimentation a lieu entre $70-80 \mathrm{~kg}$ et $110 \mathrm{~kg}$ de poids vif, celui-ci correspondant au stade commercial d'abattage. Le taux de lipides intramusculaires n'est donc pas accru après une phase de croissance compensatrice, comparati- vement à des porcs alimentés à volonté pendant la période de croissance-finition (Heyer et Lebret 2007, Lebret et al 2007). De plus, le taux de lipides intramusculaires peut même être inférieur si la restriction imposée a été sévère. Ces résultats suggèrent qu'une modification des conditions de restriction (stade de croissance et durée d'application) et de réalimentation (allongement de la durée) pourrait favoriser l'accrétion des lipides intramusculaires et ainsi améliorer la qualité sensorielle de la viande. En dehors du compartiment lipidique, la croissance compensatrice peut modifier le taux de renouvellement des protéines musculaires conduisant, dans certains cas, à une amélioration de la tendreté des viandes porcines (Kristensen et al 2004). Par contre, la qualité technologique de la viande n'est pas affectée par la croissance compensatrice (Lebret 2008).

\section{3 / Equilibre entre nu- triments, nature des nu- triments et qualité de la viande}

Outre le niveau alimentaire, la nature de l'alimentation (c'est-à-dire l'équilibre entre nutriments et la nature des nutriments), influence la composition tissulaire et par conséquent les qualités des viandes. La fraction lipidique des aliments aura une forte influence sur la composition et la valeur nutritionnelle de la viande, les autres constituants ayant assez peu d'impact. L'aspect lipidique sera donc longuement développé dans ce chapitre.

\section{1 / Equilibre entre nutri- ments : teneur en protéines et énergie de la ration}

L'effet majeur des protéines alimentaires concerne le niveau protéique de la ration et le rapport entre protéines (ou lysine, l'acide aminé le plus limitant pour la croissance) et énergie de l'aliment. Ce rapport détermine la composition du gain de poids, c'est-à-dire le dépôt de protéines et de lipides chez l'animal, donc la composition de la carcasse et des tissus musculaires et adipeux à l'abattage. Ainsi, des porcs alimentés à volonté avec un régime déficitaire en protéines ou en lysine mais adéquat en énergie présentent une augmentation des dépôts de lipides intramusculaires et en conséquence une amélioration de la tendreté et la jutosité des viandes. Cependant, ceci s'ac- 
compagne d'un accroissement significatif, bien que moins important qu'au niveau intramusculaire, de l'adiposité corporelle défavorable pour la valeur commerciale des carcasses (Castell et al 1994, Wood et al 2008).

Par contre, une réduction progressive des apports en lysine relativement à l'énergie tout au long de la phase de croissance-finition, associée à une limitation des apports en énergie, permet d'accroître spécifiquement la teneur en lipides intramusculaires jusqu'à $+40 \%$ dans le muscle Longissimus lumborum de porcs croisés Duroc, sans modifier l'épaisseur de lard dorsal et 1'adiposité corporelle (Lebret et al 2001). Cette stratégie alimentaire permet de déconnecter totalement l'accrétion des lipides au niveau intramusculaire (longe) de l'accrétion lipidique au niveau corporel (tissus sous-cutanés et internes), malgré la corrélation génétique assez forte entre ces deux caractères. Cet effet n'a pas été démontré chez le porc auparavant (figure 1). Toutefois, le fort accroissement du taux de lipides intramusculaires observé avec cette stratégie alimentaire ne s'accompagne pas d'une amélioration significative de la qualité sensorielle de la viande par rapport à la viande issue d'animaux témoins. Ceci suggère que dans la fourchette de teneur en lipides considérée dans l'étude ( 1,8 à $2,5 \%$ en moyenne), cette composante influencerait peu la qualité sensorielle.

\section{2 / Effets de la nature des protéines alimentaires sur la composition tissulaire}

La composition en acides aminés de la ration, notamment la teneur en acides aminés indispensables influence fortement la croissance des animaux et les proportions relatives de tissus maigres et gras (cf. $\S 3.1$ ). Cependant, la teneur en protéines du régime n'a pas d'impact sur celle de la viande. La composition en acides aminés des protéines musculaires dépend des proportions relatives des protéines constitutives des fibres musculaires (surtout les protéines contractiles de type actine et myosine) et des protéines de la trame conjonctive, les premières étant plus riches en acides aminés indispensables (Tomé 2008). Ainsi, la composition en acides aminés de la viande dépendra du muscle considéré (rapport protéines contractiles sur protéines conjonctives) et du traitement de la viande correspondante (notamment du type de cuisson). Il est cependant important de souligner que, de façon générale, les protéines d'origine animale (celle des viandes en particulier), sont riches en acides aminés indispensables, conférant à ces produits une valeur nutritionnelle élevée avec un apport en acides aminés approprié aux besoins de l'homme (Culioli et al 2003).

\section{3 / Effets des vitamines et des minéraux sur les constituants de la viande}

Des essais de supplémentations de l'alimentation en fer, cuivre et sélénium ont visé à améliorer les performances de croissance, l'état sanitaire de l'animal et la protection des lipides et protéines des stress oxydants. Ces apports de nutriments peuvent également modifier la teneur des minéraux de la viande. Mais très souvent, ce sont les abats qui répondent le mieux à la supplémentation, alors que le compartiment musculaire reste peu affecté (tableau 1).

Pour les deux premiers éléments, ce sont essentiellement le foie et le rein qui sont affectés. L'apport de fer n'a pas d'effet sur la flaveur de la viande, ni sur la peroxydation de ses lipides (Miller et al 1994). Pour le sélénium, la teneur peut augmenter dans les muscles et les tissus adipeux. Ceci peut donc présenter un intérêt pour faciliter l'action de la vitamine E et prévenir ainsi la peroxydation des lipides.

Comme pour les minéraux, la teneur en vitamines de la viande est en relation avec l'apport dans l'alimentation. Ceci est en particulier observé pour la vitamine $\mathrm{E}$ dont le dépôt dans la viande augmente avec la quantité ingérée : un apport supplémentaire de $150 \mathrm{mg}$ d' $\alpha$-tocophérol dans l'aliment du porc en croissance permet ainsi d'élever la teneur en vitamine $\mathrm{E}$ du muscle Longissimus lumborum de 2,9 à 4,2 $\mu \mathrm{g}$ par g de muscle (Phillips et al 2001). Outre la réduction de l'oxydation des lipides polyinsaturés et le développement d'odeurs ou goûts désagréables (rancissement) dans les viandes pen-

Tableau 1. Effet de la supplémentation en minéraux des aliments sur leurs teneurs dans la viande.

\begin{tabular}{|c|c|c|c|}
\hline Eléments & $\begin{array}{c}\text { Dose dans l'aliment } \\
(\mathrm{mg} / \mathrm{kg})\end{array}$ & $\begin{array}{c}\text { Teneur dans le foie } \\
(\mathrm{mg} / \mathrm{kg})\end{array}$ & $\begin{array}{l}\text { Teneur dans le muscle } \\
(\mathrm{mg} / \mathrm{kg})\end{array}$ \\
\hline \multirow{2}{*}{ Fer $^{(1)}$} & 60 & 100 & 9 \\
\hline & 200 & 150 & 11 \\
\hline \multirow{2}{*}{ Cuivre $^{(2)}$} & 50 & 50 & 1,5 \\
\hline & 250 & 500 & 5,5 \\
\hline Sélénium $^{(3)}$ & $\begin{array}{l}+0 \\
+05\end{array}$ & & $\begin{array}{l}0,06 \\
0,25\end{array}$ \\
\hline
\end{tabular}

D'après (1) Miller et al (1994), (2) Bradley et al (1983), (3) Mahan et Parrett (1996) dant leur conservation, la supplémentation en vitamine $\mathrm{E}$ permet d'améliorer la stabilité de la couleur et limiter la perte en eau de la viande, en limitant l'altération post mortem des membranes cellulaires très riches en acides gras polyinsaturés (Cheah et al 1995, Guo et al 2006). L'apport combiné d' $\alpha$-tocophérol et de vitamine $\mathrm{C}$ permet également de renforcer l'intensité de la couleur de la viande pour une meilleure impression visuelle (Geesink et al 2004).

\section{4 / Effets de la matière grasse de l'aliment}

Il existe une relation directe entre la composition des acides gras apportés par l'aliment du porc et celle des acides gras qui seront déposés et stockés dans la viande. Il est donc possible d'utiliser cette relation pour introduire dans l'aliment du porc des acides gras jugés bons pour la santé humaine voire indispensables pour couvrir ses besoins afin de les retrouver dans l'assiette du consommateur. Le souhait des nutritionnistes humains est de réduire la part des lipides dans notre alimentation notamment la fraction des acides gras saturés, mais aussi d'augmenter celle des acides gras polyinsaturés, en particulier ceux de la famille n-3. Deux familles d'acides gras ont fait l'objet de nombreux essais au cours des dernières années : les acides linoléiques conjugués (CLA) et les acides gras polyinsaturés $n-3$.

L'intérêt nutritionnel des CLA chez des années 90 est maintenant remis en cause. Un CLA naturel présent dans le lait (C18:2 c9 t11 ou acide ruménique) exerce un effet bénéfique sur modèles animaux (effet hypocholestérolémiant et antitumoral notamment) mais les autres formes des CLA, surtout apportés par les CLA industriels (comme le C18:2 t10 c12) ne semblent pas avoir les mêmes effets espérés vis-à-vis de la santé humaine. l'homme qui semblait prometteur fin 


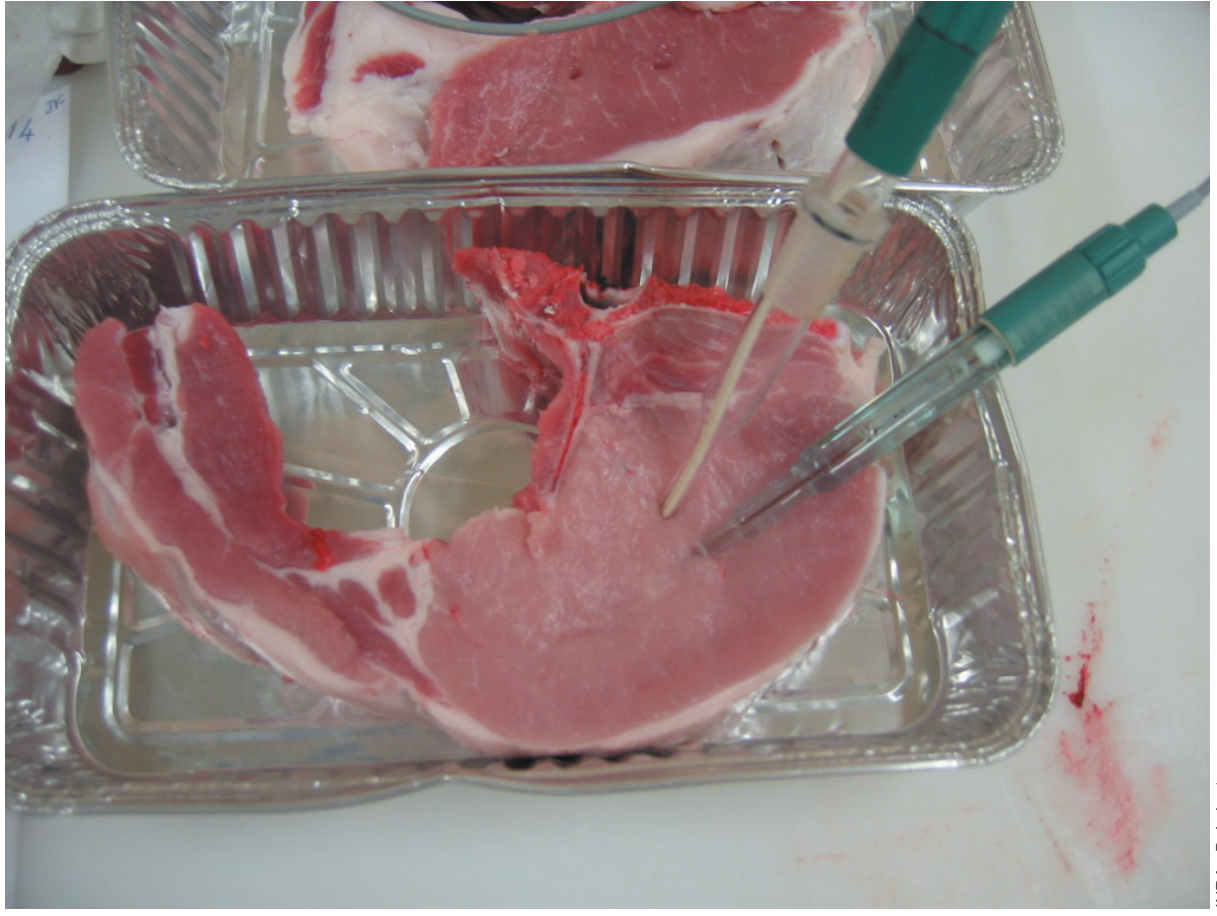

Mesure du pH ultime dans la viande.

Chez le porc, des études ont montré que la teneur en lipides des tissus adipeux était diminuée suite à une supplémentation en CLA des aliments, du fait de l'inhibition des enzymes impliquées dans la synthèse des lipides (Corino et al 2003), ce qui peut présenter un intérêt pour la qualité nutritionnelle de la viande. La teneur en CLA de la viande est également augmentée (Corino et al 2006, Bee et al 2008). Mais, face aux interrogations par rapport aux bénéfices santé réellement apportés par les CLA, et face au surcoût alimentaire induit par l'enrichissement des ali- ments en CLA, l'intérêt de l'utilisation des CLA industriels en alimentation porcine n'apparaît plus aussi souhaitable actuellement. Dans ce sens, le rôle biologique des différents CLA demande à être mieux connu.

Une autre famille d'acides gras, les acides gras polyinsaturés n-3, présente un intérêt pour la santé de l'homme. Leur rôle préventif vis-à-vis des maladies cardiovasculaires est maintenant bien établi (ANC 2001). Chez l'Homme, l'objectif est de diminuer l'ingestion des acides gras polyinsatu-

Tableau 2. Effet de l'apport de graines de lin extrudées ou d'huile de tournesol sur la composition en acides gras (expression en \% des acides gras identifiés) du muscle Longissimus et du tissu adipeux sous cutané chez le porc charcutier et le porc lourd (d'après Corino et al 2008).

\begin{tabular}{|c|c|c|c|c|c|c|c|c|}
\hline \multirow{2}{*}{$\begin{array}{c}\text { Poids vif } \\
\text { Régime }\end{array}$} & \multicolumn{4}{|c|}{$110 \mathrm{~kg}$} & \multicolumn{4}{|c|}{$160 \mathrm{~kg}$} \\
\hline & \multicolumn{2}{|c|}{ Tournesol } & \multicolumn{2}{|c|}{ Graine de lin } & \multicolumn{2}{|c|}{ Tournesol } & \multicolumn{2}{|c|}{ Graine de lin } \\
\hline & muscle & bardière & muscle & bardière & muscle & bardière & muscle & bardière \\
\hline$\Sigma A G S$ & 37,8 & 40,6 & 37,7 & 40,9 & 38,6 & 40,4 & 38,8 & 41,4 \\
\hline$\Sigma$ AGM & 44,8 & 42,4 & 44,3 & 41,6 & 48,1 & 43,1 & 46,7 & 42,8 \\
\hline$\Sigma$ AGPI & 17,4 & 17,0 & 18,0 & 17,5 & 13,3 & 16,5 & 14,5 & 15,8 \\
\hline C18:2 n-6 & 13,2 & 15,0 & 12,3 & 12,7 & 10,1 & 14,5 & 9,6 & 10,5 \\
\hline C18:3n-3 & 0,48 & 0,90 & 1,48 & 3,29 & 0,35 & 0,78 & 1,49 & 3,82 \\
\hline$C 20: 5 n-3$ & 0,12 & 0,02 & 0,36 & 0,03 & 0,07 & 0,01 & 0,39 & 0,03 \\
\hline $\mathrm{C} 22: 5 \mathrm{n}-3$ & 0,36 & 0,04 & 0,59 & 0,11 & 0,25 & 0,06 & 0,57 & 0,12 \\
\hline$C 22: 6 n-3$ & 0,07 & 0,02 & 0,14 & 0,05 & 0,06 & 0,03 & 0,09 & 0,04 \\
\hline$\Sigma$ AGPI n-6 & 16,0 & 15,4 & 15,0 & 13,4 & 12,3 & 14,9 & 11,7 & 11,3 \\
\hline$\Sigma$ AGPI n-3 & 1,02 & 0,98 & 2,57 & 3,48 & 0,72 & 0,88 & 2,53 & 4,01 \\
\hline C18:2/C18:3 & 27,6 & 17,3 & 8,3 & 3,9 & 29,4 & 18,8 & 6,5 & 2,7 \\
\hline
\end{tabular}

AGS : acides gras saturés, AGM : acides gras monoinsaturés, AGPI : acides polyinsaturés.

rés n-6 et d'augmenter celle des acides gras polyinsaturés $n-3$, une valeur du rapport de leurs précurseurs C18:2 n-6/C18:3 n-3 voisine de 5 étant souhaitée. Or, ce rapport dans l'alimentation humaine, autrefois proche de 5, est actuellement voisin de 15 à 30 (ANC 2001) suite à la modification des pratiques alimentaires en élevage ainsi qu'à l'évolution de l'alimentation humaine qui apporte de plus en plus de lipides riches en C18:2 n-6 (Ailhaud et al 2006).

De nombreuses études sont donc actuellement menées pour accroître la teneur en acides gras polyinsaturés n-3 des viandes porcines. Elles consistent essentiellement à apporter du lin dans l'alimentation mais aussi du colza et plus récemment du chanvre. Des résultats contradictoires apparaissent avec le lin, et s'expliquent par les variétés utilisées (Rousseaux 2005). Il existe, en effet, des graines de lin pauvres en matières grasses $(10 \%$ du poids frais) et en acides gras polyinsaturés n-3 (10 à $15 \%$ des acides gras totaux) et des graines de lin sélectionnées pour leur teneur très élevée en matières grasses $(50 \%)$ et riches en acides gras polyinsaturés n-3 (près de $60 \%$ des acides gras totaux). Ces dernières seront donc plus efficaces car elles apportent davantage d'acides gras polyinsaturés n-3. L'introduction de graines lin extrudées dans l'aliment du porc apparaît également plus efficace que l'huile de lin pour obtenir un dépôt plus élevé d'acides gras polyinsaturés n-3 dans la viande en raison de la meilleure digestibilité des graines (Noblet et al 2008) et d'un possible risque laxatif pour l'intestin grêle.

Des études réalisées chez le porc en croissance $(110 \mathrm{~kg})$ et le porc lourd $(160 \mathrm{~kg})$ ont montré que l'on peut augmenter fortement la teneur en acide linolénique (C18:3 n-3) dans la viande des porcs recevant des graines de lin extrudées riches en cet acide gras (Corino et al 2008). Cependant, les teneurs en acides gras à longue chaîne dérivés du précurseur C18:3 n-3, comme 1'EPA (acide eicosapentaènoïque, C20:5 n-3) et DHA (acide docosahexaènoïque, C22:6 n-3) des viandes sont peu augmentées. Ceci serait la conséquence d'une faible activité des delta 5 et 6 désaturases comme cela a été observé chez la plupart des animaux et chez l'Homme (Plourde et Cunnane 2007) (tableau 2).

L'introduction dans l'aliment du porc de DHA en grande quantité ne semble 


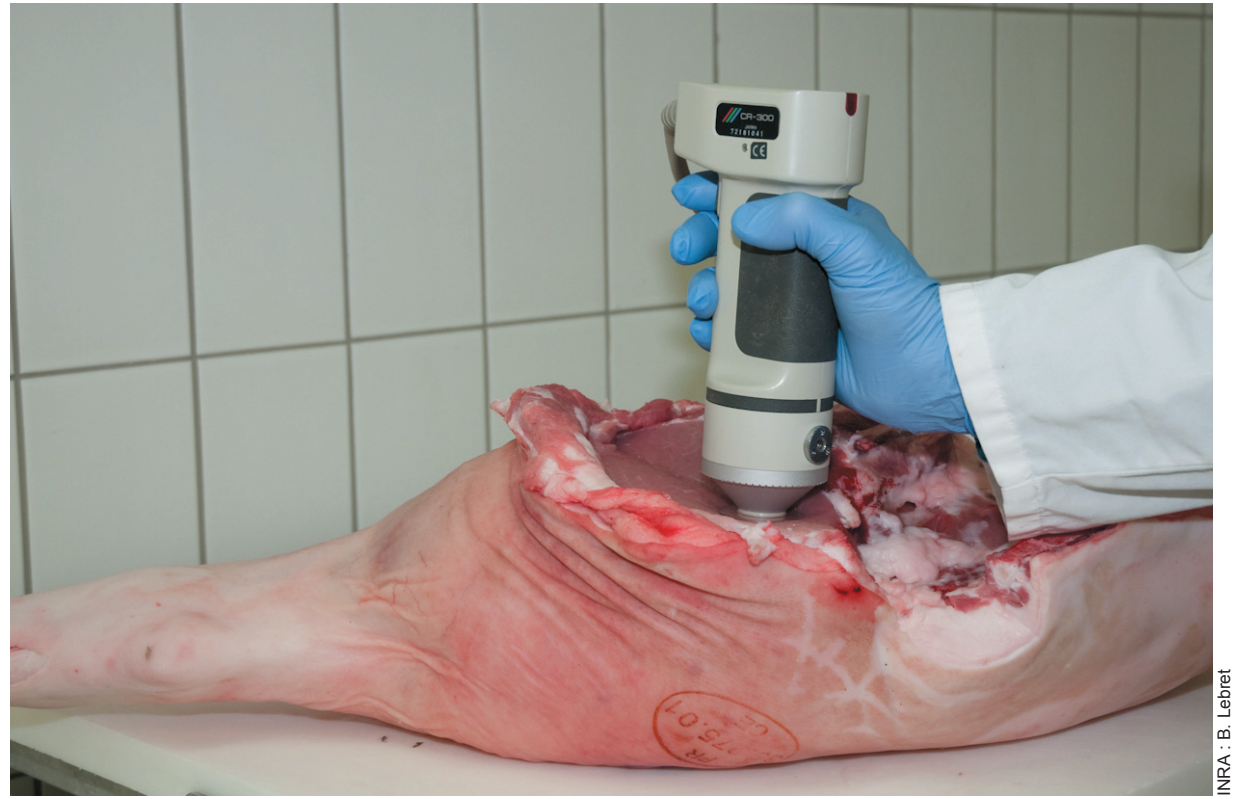

Détermination de la couleur de la viande par chromamètre.

pas donner les résultats attendus. La viande est certes enrichie en cet acide gras, mais pas autant que l'on pouvait espérer. Cet acide gras est vraisemblablement préférentiellement oxydé au niveau des peroxysomes le rendant peu disponible pour le stockage (Mourot 2009).

La qualité sensorielle des viandes enrichies en acides gras polyinsaturés n-3 peut être altérée (Kouba et al 2003). Lorsque les acides gras polyinsaturés n-3 sont apportés en faible quantité et sous forme de graines de lin (3\% de graines de lin riches en AGPI $\mathrm{n}-3$, soit $1,5 \%$ de matière grasse avec $50 \%$ d'AGPI n-3), les produits carnés sont jugés bons par les consommateurs, et même apparaissent souvent meilleurs. Les différences d'effets observés dans la viande entre graines et huiles de lin sont dues à la présence de tanins dans la graine qui protègent les acides gras de l'oxydation alors que les huiles peuvent apporter des acides gras déjà peroxydés. La consommation de viande de porc issue d'une production recevant un aliment contenant des graines de lin permet d'apporter de 350 à $450 \mathrm{mg}$ d'acide $\alpha$ linolénique pour $100 \mathrm{~g}$ de viande, ce qui représente plus du tiers des besoins préconisés par les ANC (2001). La teneur est ainsi multipliée par 5 par rapport à une viande porcine issue d'une production standard (Mourot et al 2007, Guillevic et al 2009). Ce type de production de viande enrichie en 18:3 n-3 va donc dans le sens d'une amélioration de la qualité nutritionnelle des produits souhaitée Sanitaire des Aliments (AFSSA).

\section{5 / Effets spécifiques des systè- mes de production extensifs sur la composition tissulaire et la qualité de la viande}

Les systèmes de production des animaux influencent la composition tissulaire et la qualité des viandes via les effets combinés de l'alimentation (niveau, nature des nutriments), des types et activité des animaux (génotype, âge, sexe, exercice physique) et de l'environnement (type d'habitat, conditions climatiques...) sur la vitesse et la composition des dépôts musculaires et adipeux (Lebret 2008). Concernant l'alimentation, il faut noter la particularité des systèmes de production extensifs dans lesquels tout ou partie de la ration alimentaire est constituée des ressources naturelles variées produites sur les parcs ou zones d'engraissement des animaux telles que l'herbe, les glands et/ou les châtaignes employées pour les productions extensives méditerranéennes. Ces types d'élevage et d'alimentation modifient fortement la composition des tissus à l'abattage et la qualité ultérieure des produits. Ainsi, en élevage plein air, la consommation d'herbe en complément de la distribution d'aliment conventionnel conduit à un dépôt important d'acides gras polyinsaturés n-3 (notamment C18:3 n-3) et d'antioxydants d'origine végétale ( $\alpha$ et par l'Agence Française de Sécurité $\gamma$-tocophérols) dans les tissus musculaires et adipeux chez le porc en croissance comme la truie adulte, améliorant ainsi le statut nutritionnel des viandes. Par exemple, le rapport en AGPI n-6/AGPI n-3 est réduit de près de $50 \%$ (de 10,3 à 5,7) dans le muscle Longissimus de truies de réforme élevées en plein air ayant accès au pâturage, relativement à l'élevage des mêmes truies en claustration (Nilzen et al 2001, Lebret et Guillard 2005). Dans les systèmes de production traditionnels méditerranéens, la consommation importante de glands ou de châtaignes et d'herbe par les porcs, seules ressources alimentaires offertes en période de finition, conduit à une croissance compensatrice caractérisée par une accrétion lipidique très forte aux niveaux corporel et intramusculaire. Dans ce cas, le système d'élevage, notamment la conduite alimentaire, permet aux animaux (races locales) d'exprimer leur fort potentiel de dépôt de lipides, notamment au niveau intramusculaire. Le profil en acides gras des tissus est également modifié avec une augmentation importante des acides gras monoinsaturés (notamment le C18:1 n-9cis d'origine alimentaire ou synthétisé par l'animal) et polyinsaturés (C18:3 n-3, C22:5 n-3 et C22:6 n-3 d'origine alimentaire). De même, les teneurs en antioxydants tissulaires sont fortement accrues suite à leur concentration élevée dans les ressources alimentaires. $\mathrm{Au}$ final, les teneurs et la nature des lipides et des micronutriments antioxydants des produits sont notablement modifiées, conférant des qualités sensorielles et nutritionnelles spécifiques aux produits issus de ces systèmes (Lebret 2008).

\section{Conclusion}

Parmi les facteurs d'élevage, l'alimentation peut influencer fortement les qualités sensorielles et nutritionnelles de la viande de porc. Les orientations actuelles de production ne doivent plus prendre en compte simplement la quantité de viande produite mais aussi la qualité des produits. Cette dernière doit être définie en relation avec les transformateurs et les nutritionnistes sans oublier les attentes des consommateurs. Une meilleure information sur la valeur nutritionnelle doit aussi être poursuivie auprès des consommateurs. 


\section{Références}

Ailhaud G., Massiera F., Weill P., Legrand P., Alessandri J.M., Gusnet P., 2006. Temporal changes in dietary fats: role of n-6 polyunsaturated fatty acids in excessive adipose tissue development and relationship to obesity. Prog. Lipid Res., 45, 203-236.

ANC, 2001. Apports Nutritionnels Conseillés pour la population française. AFSSA, Ed. Tec \& Doc, Paris, France.

Bee G., Jacot S., Guex G., Biolley C., 2008. Effects of two supplementation levels of linseed combined with CLA or tallow on meat quality traits and fatty acid profile of adipose and different muscle tissues in slaughter pigs. Animal, 2, 800-811.

Bradley B.L., Graber G., Condon R.J., Frobish L.T., 1983. Effects of graded levels of dietary copper on copper and iron concentrations in swine tissues. J. Anim. Sci., 56, 625-630.

Brewer M.S., Zhu L.G., McKeith F.K., 2001. Marbling effects on quality characteristics of pork loin chops: consumer purchase intent, visual and sensory characteristics. Meat Sci., 59, 153-163.

Castell A.G., Cliplef R.L., Poste-Flynn L.M., Butler G., 1994. Performance, carcass and pork characteristics of castrates and gilts self-fed diets differing in protein content and lysine: energy ratio. Can. J. Anim. Sci., 74, 519-528.

Cheah K.S., Cheah A.M., Krausgrill D.I., 1995. Effect on dietary supplementation of vitamin E on pig meat quality. Meat Sci., 39, 255264.

Corino C., Magni S., Pastorelli G., Rossi R, Mourot J., 2003. CLA in heavy pig nutrition: influence on growth, meat quality and dry-cured ham sensory characteristics. J. Anim. Sci., 81, 2219-2229.

Corino C., Pastorelli G., Douard V., Rossi R., Musella M., Mourot J., 2006. L'acide linoléique conjugué (ALC ou CLA) en nutrition porcine. INRA Prod. Anim., 19, 39-46.

Corino C., Musella M., Mourot J., 2008. Influence of extruded linseed on growth, carcass composition, and meat quality of pigs slaughtered at 110 and $160 \mathrm{~kg}$ liveweight. J. Anim. Sci., $86,1850-1860$

Culioli J., Berri C., Mourot J., 2003. Muscle foods: consumption, composition and quality. Sciences des Aliments, 23, 13-34.

Ellis M., Webb A.J., Avery P.J., Brown I., 1996. The influence of terminal sire genotype, sex, slaughter weight, feeding regime and slaughter-house on growth performance and carcass and meat quality in pigs and on the organoleptic properties of fresh pork. Anim. Sci., 62, 521-530.
Fernandez X., Monin G., Talmant A., Mourot J., Lebret B., 1999a. Influence of intramuscular fat content on the quality of pig meat. 1 . Composition of the lipidic fraction and sensory characteristics muscle longissimus lumborum. Meat Sci., 53, 59-65.

Fernandez X., Monin G., Talmant A., Mourot J., Lebret B., 1999b. Influence of intramuscular fat content on the quality of pig meat. 2 . Consumer acceptability of muscle longissimus lumborum. Meat Sci., 53, 67-72.

Geesink G.H., van Buren R.G.C., Savenije B. Verstegen M.W.A., Ducro B.J., van der Palen J.P.G., 2004. Short-term feeding strategies and pork quality. Meat Sci., 67, 1-6.

Guillevic M., Kouba M., Mourot J, 2009. Effect of a linseed diet on lipid composition, lipid peroxidation and consumer evaluation of fresh meat and French cooked pork meats. Meat Sci., accepté.

Guo Q., Richert B.T., Burgess J.R., Webel D.M., Orr D.E., Blair M., Grant A.L., Gerrard D.E., 2006. Effect of dietary vitamin E supplementation and feeding period on pork quality. J. Anim. Sci., 84, 3071-3078.

Heyer A., Lebret B., 2007. Compensatory growth response in pigs: Effects on growth performance, composition of weight gain at carcass and muscle levels, and meat quality. J. Anim. Sci., 85, 769-778.

Kouba M., Enser M., Whittington F.M., Nute G.R.,Wood J.D., 2003. Effect of a high-linolenic acid diet on lipogenic enzyme activities, fatty acid composition, and meat quality in the growing pig. J. Anim. Sci., 81, 1967-1979.

Kristensen L., Therkildsen M., Aaslyng M.D. Oksbjerg N., Ertbjerg P., 2004. Compensatory growth improves meat tenderness in gilts but not in barrows. J. Anim. Sci., 82, 3617-3624.

Lebret B., 2004. Conséquences de la rationalisation de la production porcine sur les qualités des viandes. INRA Prod. Anim., 17, 79-91.

Lebret B., 2008. Effects of feeding and rearing systems on growth, carcass composition and meat quality in pigs. Animal, 2, 1548-1558.

Lebret B., Guillard A.S., 2005. Outdoor rearing of cull sows: effects on carcass, tissue composition and meat quality. Meat Sci., 70, 247257.

Lebret B., Juin H., Noblet J., Bonneau M., 2001. The effects of two methods of increasing age at slaughter on carcass and muscle traits and meat sensory quality in pigs. Anim. Sci., 72, 87-94.

Lebret B., Heyer A., Gondret F., Louveau I., 2007. The response of various muscle types to a restriction-realimentation feeding strategy in growing pigs. Animal, 1, 849-857.
Mahan D.C., Parrett N.A., 1996. Evaluating the efficacy of selenium-enriched yeast and sodium selenite on tissue selenium retention and serum glutathione peroxidase activity in growe and finisher swine. J. Anim. Sci. 74, 2967-2974.

Miller M.A., Shaw A., Kraut J., 1994. A structure of oxy-peroxidase as a model for the transient enzyme: peroxide complex. Nat. Struct. Biol., 1, 524-531.

Mourot J., 2009. Sensorial and nutritiona quality of pork. In: Improving the sensory and nutritional quality of fresh meat: New technologies. Kerry J.P., Ledward D.A. (Eds), Woodhead Publishing, Cambridge, England. 342-355.

Mourot J., Guillevic M., Blochet J.E., Le Minous A.E., 2007. Lutter contre les croyances une nouvelle évaluation de l'intérêt nutritionnel des charcuteries. Rev. Nutr. Prat., 19, 20-26.

Nilzen V., Babol J., Dutta P.C., Lundeheim N., Enfält A.C., Lundström K., 2001. Free range rearing of pigs with access to pasture grazing effect on fatty acid composition and lipid oxidation products. Meat Sci., 58, 267-275.

Noblet J., 2005. Protein and energy requirements of growing swine. Proc. II Simp. Int. sobre exigencias nutricionais de a ves e suinos. Rostagno H., Albina L. (Eds), 175-198.

Noblet J., Jaguelin-Peyraud Y., Quemeneur B., Chesneau G., 2008. Valeur énergétique de la graine de lin chez le porc : impact de la technologie de caisson-extrusion. Journ. Rech. Porcine Fr., 40, 203-208.

Phillips A.L., Faustman C., Lynch M.P., Govoni K.E., Hoagland T.A., Zinn S.A., 2001 Effect of dietary $\alpha$-tocopherol supplementation on color and lipid stability in pork. Meat Sci., 58, 389-393.

Plourde M., Cunnane S.C., 2007. Extremely limited synthesis of long chain polyunsaturates in adults: implications for their dietary essentiality and use as supplements Appl. Physiol. Nutr. Metabol., 32, 619-634.

Rousseaux E., 2005. Le lin et ses secrets, Geste Editions, La Crèche, France, 56p.

Tomé D., 2008. Qualité nutritionnelle des protéines de la viande. Cah. Nutr. Diét., 43, Hors Série 1, 1S40-1S45.

Volatier J.L., 2000. Enquête INCA (Individuelle et Nationale sur Consommations Alimentaires), Coll. AFSSA, 280p.

Wood J.D., Enser M., Fisher A.V., Nute G.R Sheard P.R., Richardson R.I., Hughes S.I., Whittington F.M., 2008. Fat deposition, fatty acid composition and meat quality: a review. Meat Sci., 78, 343-358

\section{Résumé}

Parmi les facteurs d'élevage influençant la qualité de la viande chez le porc, l'alimentation joue un rôle central. Le niveau et le profil de distribution de la ration (restriction puis réalimentation) influencent la vitesse et la composition du gain de poids (protéines/lipides) aux niveaux corporel et tissulaire, et, donc, la composition de la carcasse et des tissus. La qualité sensorielle de la viande peut être ainsi modifiée, notamment via la teneur en lipides intramusculaires. L'équilibre entre les principaux nutriments, notamment les protéines et l'énergie de la ration, conditionnent également la vitesse et la nature des dépôts tissulaires, pouvant ainsi affecter la qualité sensorielle des viandes. La nutrition constitue un puissant levier pour modifier le profil lipidique des viandes porcines, la teneur en acides gras (polyinsaturés n-3 notamment), et par suite leur valeur nutritionnelle. 


\begin{abstract}
Effects of pig diet on the quality of pork and pork products

Among pig rearing conditions, feeding highly influences the quality of pork and pork products. The feeding level and profile (restrictionrealimentation) determine the rate and composition of growth (lipids/proteins) at both body and tissue levels, thereby influencing carcass and tissue composition. Thus, the eating quality of pork may be modified as a consequence of variation in intramuscular fat content. The balance between main nutrients, in particular the protein and energy levels, also influences growth rate and tissue composition. In pigs, the lipid composition of tissues highly depends on the lipid composition of the diet. Therefore, the fatty acid profile of meat (n-3 PUFA content for example) can be easily modified through feeding, thereby improving the quality of pork for the consumer and meeting the nutritionist's requirements.
\end{abstract}

MOUROT J., LEBRET B., 2009. Modulation de la qualité de la viande de porc par l'alimentation. Inra Prod. Anim., 22, $33-40$. 
